BUHEP-99-12

MADPH-99-1120

SHEP-99-05

\title{
Bounds on Flavor Gauge Bosons from precision Electroweak Data
}

\author{
Gustavo Burdman* \\ Department of Physics, University of Wisconsin, Madison, WI 53706, USA. \\ R. Sekhar Chivukula ${ }^{\dagger}$ \\ Department of Physics, Boston University, Boston, MA 02215, USA. \\ Nick Evans \\ Department of Physics, University of Southampton, Southampton, S017 1BJ, UK.
}

June 1999

\begin{abstract}
Gauged flavor symmetries at low energies have been proposed in models of dynamical electroweak symmetry breaking and fermion mass generation. The massive flavor gauge bosons give rise to corrections to precisely measured electroweak quantities. We perform a fit to the collider electroweak data and place indirect limits on such new physics. In particular we study several models from the literature: universal coloron; chiral top color; chiral quark family symmetry; SU(9) and SU(12) chiral flavor symmetry. The $95 \%$ exclusion limits on the mass of the heavy gauge bosons for these models, at their critical coupling for chiral symmetry breaking, typically lie between 1-3 TeV. We discuss the robustness of these bounds with respect to changes in higgs mass, b-quark asymmetry data, the SLD left right asymmetry data, or additional new physics.
\end{abstract}

\footnotetext{
*burdman@pheno.physics.wisc.edu

†sekhar@bu.edu

${ }^{\ddagger}$ n.evans@phys.soton.ac.uk
} 


\section{Introduction}

The Standard Model (SM), in the limit where we neglect all gauge interactions and fermion masses, has a large SU(45) global symmetry corresponding to the fact that in this limit there are 45 chiral fermion fields that are indistinguishable. The gauge interactions of the SM are by necessity subgroups of this maximal symmetry, they are in particular the familiar $S U(3)_{c} \times S U(2)_{L} \times U(1)_{Y}$ gauge symmetry. There is no current theoretical understanding of why this particular subgroup is picked out to be gauged. Furthermore we have learned from the SM that gauge symmetries may be broken and not manifest at low energies. The possibility therefore exists that at high energies a larger subgroup of the SU(45) symmetry is gauged, and then broken by some dynamics or higgs mechanism to the SM gauge group.

These gauged flavor symmetries have been invoked in a number of scenarios to play a role in the dynamical generation of fermion masses. For example they may play the part of extended technicolor [1] interactions in technicolor models [2] or top condensation models [3], feeding the electroweak symmetry (EWS) breaking fermion condensate down to provide masses for the lighter standard model fermions. Strongly interacting flavor gauge interactions may also be responsible for the condensation of the fermions directly involved in EWS breaking. For example, top condensation has been postulated to result from a topcolor gauge group [4] and, in the model of ref. [5], from family gauge interactions. There has been renewed interest in these models recently with the realization that variants, in which the top mixes with singlet quarks, can give rise to both the EW scale and an acceptable top mass via a seesaw mass spectrum [6]. These top seesaw models have the added benefit of a decoupling limit which allows the presence of the singlet fields to be suppressed in precision EW measurements, bringing these dynamical models in line with the data. Flavor universal variants [7] with the dynamics driven by family or large flavor gauge symmetries have also been constructed.

The naive gauging of flavor symmetries at low scales (of order a few TeV) often gives rise to unacceptably large flavor changing neutral currents (FCNC) since gauge and mass eigenstates do not coincide (gauge symmetries that give rise to direct contributions to $K^{0}-$ $\bar{K}^{0}$ mixing typically are constrained to lie above $500 \mathrm{TeV}$ in mass scale). There are, though, many models that survive these constraints. Gauge groups that only act on the third family are less experimentally constrained - topcolor is such an example. Models in which the chiral flavor symmetries of the SM fermions are gauged preserving the SM $U(3)^{5}$ flavor symmetry [8] respect the GIM mechanism even above the breaking scale and do not give rise to tree level FCNCs [9]. There are also strong constraints on gauged flavor models where the dynamics responsible for the breaking of the flavor symmetry does not respect custodial isospin [10]. We shall restrict ourselves to models where the top mass is the sole source of custodial isospin 
breaking.

Since these interactions may exist at relatively low scales (a few $\mathrm{TeV}$ ), where they do not completely decouple, and play an integral part in either EWS breaking or fermion mass generation, it is interesting to study the current experimental bounds. In this paper we will concentrate on the bounds from precision EW measurements [11]. We will constrain a number of models that have been proposed in this context: the universal coloron model [12]; chiral top color [6]; chiral quark family gauge symmetry [9, 5, 7]; SU(9) chiral quark flavor symmetry [13, [7]; and SU(12) chiral, flavor symmetry [13, 7]. Except in the last section, for simplicity the only fermions included in our analysis are the ordinary quarks and leptons. Therefore, as presented here the models will not be anomaly free. The models can be made anomaly free by the introduction of additional fermions that obtain masses at or above the gauge boson mass scale as a result of the flavor symmetry breaking sector. The SM fermions that are light below that scale survive because they are chiral under the remaining, unbroken gauge interactions - this is an example of Georgi's "extended survival hypothesis" [14. For each of the gauge symmetries we study, an explicit example of an anomaly-free model exists in the literature in the references provided above.

The gauge bosons can enter into precision EW predictions either through corrections to the $Z$ mass from isospin violating effects in top loops [15] and mixing with the $Z$ [10], or as vertex corrections to the $Z$-fermion vertices [16]. We review the generic form of these corrections in Section 2. In Section 3 we describe our fit to the $Z$-pole data. In Section 4 we review each of the models under study and display the precision limits. To this point the limits are on the models with only the flavor gauge bosons as new physics. The contributions to the $\rho$ parameter are a significant constraint and our limits correspond to the hard limit on $\Delta \rho($ or $\alpha T)$ of $\leq 0.1 \%$ [17]. The softer upper bound on $\Delta \rho$ often quoted of $0.4 \%$ [17] assumes the existence of extra new physics contributing to the $\mathrm{S}$ parameter 18 to maximize the possible value of $\Delta \rho$. The flavorons do not contribute to $\mathrm{S}$ (at leading order) and so the harder limit is applicable here. In Section 5 we discuss the dependence of the limits on changes to the higgs mass. The limits are largely insensitive except in the case of models which give rise to large corrections to the $\Delta \rho / \mathrm{T}$ parameter (in particular the coloron models) and prefer a heavy higgs in order to cancel that contribution. The bounds on these models decrease for higgs masses as large as $650 \mathrm{GeV}$ which, without the flavorons, would be ruled out at $95 \%$ confidence in the SM. We also discuss the dependence of the limits on the b quark asymmetries which are the measurements with the most deviation from the SM predictions, and the SLD measurement of the left right asymmetry which provides one of the strongest constraints on the higgs mass.

It is important to remember the limitation of indirect constraints such as these; additional 
unanticipated new physics may exist that enters the fit and in principle may cancel all contributions from the gauge bosons, completely removing any bound (or equally the bound may be considerably toughened by extra new physics)! In practice such a conspiracy seems unlikely and the bounds we calculate provide a sensible guide to the exclusion limit. As an example of the possible effect of additional new physics, in Section 5 we also discuss the addition of vertex corrections from mixing between the SM fermions and singlet massive fermions such as occurs in the top [6] and flavor universal [7] see-saw models. Another possible source of $\mathrm{S}$ and $\mathrm{T}$ contributions are the scalar sectors of these models, which are potentially light. However, enumerating these scalars is model dependent and their masses are hard to estimate reliably so we do not attempt to include them. We expect the few $\mathrm{TeV}$ bound on the flavoron masses coming from our fit to hold even with such perturbations.

\section{Flavoron Corrections to EW Parameters}

The massive flavor gauge bosons (flavorons) under study give a number of corrections to low energy EW parameters. In this section we provide generic calculations of these effects.

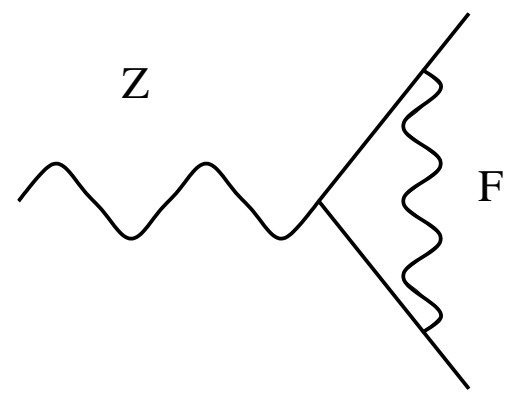

Figure 1: $Z$ vertex corrections from heavy flavorons.

Flavorons acting on the SM fermions give rise to vertex corrections in the low energy SM. We assume that there are no massive fermions beyond those in the SM transforming under the flavor gauge groups as part of the same gauge multiplet as the SM fermions (an example would be techni-fermions). These would give rise to overly large corrections, as is well known from extended technicolor models [19]. The main corrections to $Z$ vertices occur at order $p^{2}$ in the expansion of the penguin vertex correction in Fig 1. Hill and Zhang [16] have calculated the correction to the vertex to be

$$
\Delta g_{f}=g_{f} \frac{G \kappa_{F}}{6 \pi} \frac{M_{Z}^{2}}{M_{F}^{2}} \ln \left(\frac{M_{F}^{2}}{M_{Z}^{2}}\right)
$$


where $g_{f}$ is the coupling for the $Z$-fermion vertex, $G$ is the appropriate group theory factor for the diagram, $\kappa_{F}=g_{F}^{2} / 4 \pi$ with $g_{F}$ the flavoron gauge coupling, and $M_{F}$ the flavoron mass.

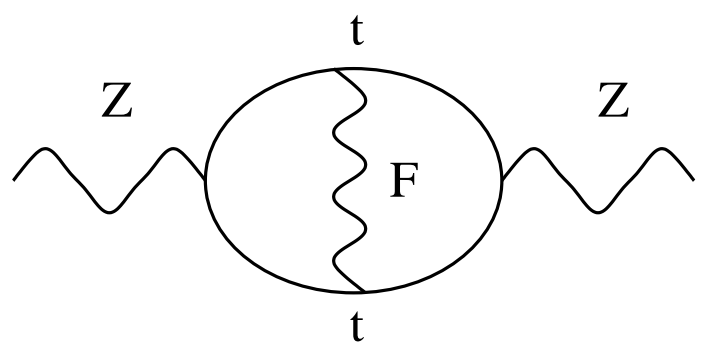

Figure 2: Flavoron corrections to the $Z$ mass through top loops.

The flavorons can also give corrections to the $Z$ mass through the diagram in Fig 2 involving a top loop [15]. The top bottom mass splitting provides the source of isospin breaking so there are contributions to $\Delta \rho(T)$ even when the flavoron physics is isospin preserving.

The contribution to $\mathrm{T}$ is

$$
T=\frac{\pi}{s_{\theta_{w}}^{2} c_{\theta_{w}}^{2} M_{Z}^{2}}\left[2 \Pi_{L L}\left(m_{t}, 0\right)-\Pi_{L L}\left(m_{t}, m_{t}\right)\right]
$$

where $\Pi_{L L}\left(m_{t}, 0\right)$ and $\Pi_{L L}\left(m_{t}, m_{t}\right)$ refer to the left-handed vacuum polarizations involving one top and one (massless) bottom quark, and two top quarks in the loop respectively, and they are evaluated at zero momentum transfer. Following 15 we will approximate the two loop diagram by a product of two one loop diagrams after the flavoron propagator has been contracted to a point interaction.

Allowing the flavoron to have different couplings to left and right handed top quarks we find

$$
\begin{aligned}
\Pi_{L L}\left(m_{t}, 0\right) & =-\frac{N_{c} G_{L L}^{\prime}}{64 \pi^{3}} m_{t}^{4}\left[\ln \left(M_{F}^{2} / m_{t}^{2}\right)\right]^{2} \frac{\kappa_{F}}{M_{F}^{2}} \\
\Pi_{L L}\left(m_{t}, m_{t}\right) & =-\frac{N_{c}\left(G_{L L}^{\prime}+G_{R R}^{\prime}\right)}{16 \pi^{3}} m_{t}^{4}\left[\ln \left(M_{F}^{2} / m_{t}^{2}\right)\right]^{2} \frac{\kappa_{F}}{M_{F}^{2}}
\end{aligned}
$$

with $G_{L L}^{\prime}$ and $G_{R R}^{\prime}$ appropriate group theory factors for the model for the interaction between two left handed quarks and two right handed quarks respectively, and where we have evaluated the leading logarithm. The resulting contribution to $\mathrm{T}$ is

$$
T=N_{c}\left(G_{L L}^{\prime}+2 G_{R R}^{\prime}\right) \frac{m_{t}^{4}}{32 \pi^{2} s_{\theta_{w}}^{2} c_{\theta_{w}}^{2} M_{Z}^{2}} \frac{\kappa_{F}}{M_{F}^{2}}\left[\ln \left(M_{F}^{2} / m_{t}^{2}\right)\right]^{2}
$$




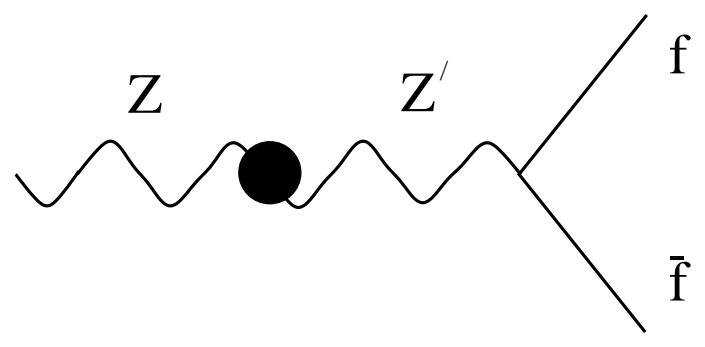

Figure 3: Flavoron- $Z$ mixing shift to SM couplings.

There is a final possible source of contributions to the $Z$ vertex corrections and the $T$ parameter in models where a generator of the flavor group (we will call the associated gauge boson $Z^{\prime}$ ) mixes with hypercharge or $T_{3}$ of the weak $\mathrm{SU}(2)$ group [10]. If at the weak scale the $Z Z^{\prime}$ mass matrix takes the form

$$
\left(Z^{\mu} Z^{\prime \mu}\right)\left(\begin{array}{cc}
M_{Z}^{2} & \Delta M^{2} \\
\Delta M^{2} & M_{F}^{2}
\end{array}\right)\left(\begin{array}{c}
Z_{\mu} \\
Z_{\mu}^{\prime}
\end{array}\right),
$$

and if $M_{F}^{2} \gg M_{Z}^{2}, \Delta M^{2}$ then there will be corrections to the $Z$-fermion couplings in the low energy theory of the form (from Fig 3)

$$
\Delta g_{f} \simeq-\frac{\Delta M^{2}}{M_{F}^{2}} g_{f}^{Z^{\prime}}
$$

and, diagonalizing the mass matrix so that the shift in the low energy $Z$ mass is

$$
\Delta M_{Z}^{2}=-\frac{\left(\Delta M^{2}\right)^{2}}{M_{F}^{2}}
$$

gives a $\mathrm{T}$ parameter contribution

$$
T=+\frac{\Delta M_{Z}^{2}}{\alpha M_{Z}^{2}}
$$

Clearly for these contributions to be small we require that the only sources of custodial isospin breaking occur at the weak scale so $\Delta M$ is of order $v$ and not of order $M_{F}$. The models we consider all respect custodial isospin at the flavor symmetry breaking scale.

These shifts in low energy SM parameters are the leading corrections. The shifts in the $Z$-fermion vertices will for example also give rise to $\mathrm{S}$ parameter contributions at loop level 222 but these are considerably smaller than the direct effects and we neglect them. 


\section{Fitting To Precision Data}

To place bounds on the models we use primarily the $Z$ pole precision observables from the LEP and SLD experiments plus the collider measurements of the $\mathrm{W}$ mass. We use the data and SM fit from [11]

\begin{tabular}{c|ccc}
$V_{i}$ & Tree Level & Measurement & SM fit pull \\
\hline$\Gamma_{Z}(G e V)$ & $\Gamma_{l e p} \Gamma_{h a d} / \Gamma_{Z}$ & $2.4939 \pm 0.0024$ & -0.80 \\
$\sigma_{0}$ & $\Gamma_{h a d} / \Gamma_{l e p}$ & $41.491 \pm 0.058$ & 0.31 \\
$R_{l}$ & $N_{c}\left(g_{b_{L}}^{2}+g_{b_{R}}^{2}\right) / \Gamma_{h a d}$ & $0.21656 \pm 0.00074$ & 0.90 \\
$R_{b}$ & $N_{c}\left(g_{c_{L}}^{2}+g_{c_{R}}^{2}\right) / \Gamma_{h a d}$ & $0.1735 \pm 0.0044$ & 0.29 \\
$R_{c}$ & $\left(g_{e_{L}}^{2}-g_{e_{R}}^{2}\right) /\left(g_{e_{L}}^{2}+g_{e_{R}}^{2}\right)$ & $0.1479 \pm 0.0051$ & 0.25 \\
$A_{e}$ & $\left(g_{b_{L}}^{2}-g_{b_{R}}^{2}\right) /\left(g_{b_{L}}^{2}+g_{b_{R}}^{2}\right)$ & $0.867 \pm 0.035$ & -1.93 \\
$A_{b}$ & $\left(g_{c_{L}}^{2}-g_{c_{R}}^{2}\right) /\left(g_{c_{L}}^{2}+g_{c_{R}}^{2}\right)$ & $0.647 \pm 0.040$ & -0.52 \\
$A_{c}$ & $3 A_{e} A_{b} / 4$ & $0.0990 \pm 0.0021$ & -1.81 \\
$A_{f b}^{0, b}$ & $3 A_{e} A_{c} / 4$ & $0.0709 \pm 0.0044$ & -0.58 \\
$A_{f b}^{0, c}$ & $3 A_{e}^{2} / 4$ & $0.01683 \pm 0.00096$ & 0.73 \\
$A_{f b}^{0, e}$ & $\left(g_{\tau_{L}}^{2}-g_{\tau_{R}}^{2}\right) /\left(g_{\tau_{L}}^{2}+g_{\tau_{R}}^{2}\right)$ & $0.1431 \pm 0.0045$ & -0.79 \\
$A_{\tau}$ & $A_{e}$ & $0.1510 \pm 0.0025$ & 1.75 \\
$A_{L R}$ & $M_{W}^{S M}(1+0.0055 T-0.0036 S)$ & $80.37 \pm 0.09$ & -0.01 \\
$M_{W}^{L E P 2}$ & $M_{W}^{S M}(1+0.0055 T-0.0036 S)$ & $80.41 \pm 0.09$ & 0.43 \\
$M_{W}^{p p}$ & $M_{W}^{S M}$ & &
\end{tabular}

where

$$
\begin{gathered}
\Gamma_{Z}=\sum_{f}\left(g_{f_{L}}^{2}+g_{f_{R}}^{2}\right) \\
\Gamma_{h a d}=\sum_{h a d}\left(g_{h_{L}}^{2}+g_{h_{R}}^{2}\right) \\
\Gamma_{\text {lep }}=\sum_{\text {lep }}\left(g_{l_{L}}^{2}+g_{l_{R}}^{2}\right)
\end{gathered}
$$

The top quark, of course, does not contribute to any Z-pole variables at tree level.

We use the fit of [11] as our test Standard Model into which we introduce corrections [21]. The full fit of [11] includes measurements of the $\mathrm{Z}$ mass and $\sin ^{2} \theta_{W}$ as determined in low energy neutrino scattering data. For simplicity, in comparing the predictions of our models to electroweak data we use the $\mathrm{Z}$ mass (the most precisely measured quantity) as a (fixed) input to our calculation, and do not include $\sin ^{2} \theta_{W}$.

As an illustration of the correspondence of our fit to that given in [11], we may compare the constraints on the higgs mass in the standard model. The fit of [11] corresponds to a best fit higgs mass of $m_{h}=76 \mathrm{GeV}$, and a bound of $262 \mathrm{GeV}$ at $95 \%$ confidence level. Variation in the higgs mass may be viewed as contributions to the $\mathrm{S}$ and $\mathrm{T}$ parameters which 
are calculated in [20]. Using this method to incorporate variations in the higgs mass and with the more limited set of precision variables we consider, we find a $95 \%$ confidence limit upper bound of $230 \mathrm{GeV}(120 \mathrm{GeV}$ at $67 \%)$, in good agreement with the full fit.

Furthermore, because the higgs mass enters precision measurements only logarithmically, the limit on the higgs mass is very sensitive to small changes in the measured parameters and the difference in the higgs mass bound exaggerates the difference between the two fits. In contrast, the flavoron masses enter quadratically in corrections to precision electroweak observables, and the bounds we derive would not change significantly were we to incorporate $M_{Z}$ and $\sin ^{2} \theta_{W}$. For definiteness, we will take a higgs mass of $100 \mathrm{GeV}$ as our standard in the fits of section 4 . In Section 5 we show that the bounds are largely insensitive to changes in the higgs mass.

The flavoron models affect the low energy predictions through the $Z$-fermion coupling shifts described in Section 2. The leading order shifts as a function of the model parameters $\delta g_{i}\left(\kappa, M_{F}\right)$ are easily calculable from the tree level expressions in (10) for the electroweak observables $\left(M_{W}\right.$ is independent of the shifts to the Z-fermion couplings). The models also give rise to shifts in the $\Delta \rho(T)$ parameter.

We include one non- $Z$-pole measurement in our fit, the lattice determination of $\alpha_{s}$ [17]

$$
\alpha_{s}^{\text {lattice }}\left(M_{Z}\right)=0.117 \pm 0.003
$$

This constraint is important for our fit since the vertex corrections in some of our models (in particular the universal coloron model) mimic a shift in $\alpha_{s}\left(M_{Z}\right)$. Fitting to the lattice data ensures we do not produce an unacceptable value for $\Lambda_{Q C D}$. The value of $\alpha_{s}\left(M_{Z}\right)$ is, by these standards, imprecisely determined and therefore we include possible variation in the input parameter $\alpha_{s}\left(M_{Z}\right)$ through $\delta \alpha_{s}$, a parameter which we will include in the fit. We include the effect $\delta \alpha_{s}$ on electroweak measurements by modifying the quark copulings

$$
\delta g_{q}=\frac{e}{s_{\theta} c_{\theta}}\left(T_{3}-Q s_{\theta}^{2}\right) \frac{\delta \alpha_{s}}{2 \pi}
$$

We perform the fit as follows. For a given model and choice of $M_{F}$ and $m_{h}$ we have predictions for the shifts in the SM quantities in (10) as a function of $\kappa_{F}$ and $\delta \alpha_{s}$. We calculate $\chi^{2}$ and place a limit on $\kappa_{F}$ by finding the $95 \%$ confidence value of $\chi^{2}$ allowing $\delta \alpha_{s}$ to take a value that minimizes $\kappa_{F}$. The number of degrees of freedom is 14 - the number of fitted variables (16) minus 2 for the 2 fitted quantities.

\footnotetext{
${ }^{1}$ The leading order QCD correction to the hadronic branching ratios are $\Gamma_{h}=\Gamma_{h}^{0}\left(1+\alpha_{s} / \pi\right)$.
} 


\section{Models and Limits}

We present five models as examples of flavoron physics and give their limits from the EW precision fit.

\subsection{Universal Coloron}

The universal coloron model [12] at high energies has the gauge symmetry of the SM with regards the SM fermions but, in addition, an extra $\mathrm{SU}(3)$ gauge group acting on a new fermion or scalar sector. That new sector also transforms under the proto-color group. It is assumed that dynamics occurring in the new sector makes all the additional matter massive and induces a vev $(V)$ for an effective higgs in the $(3, \overline{3})$ of the proto-color group and the additional $\mathrm{SU}(3)$. The two sets of $\mathrm{SU}(3)$ gauge bosons mix through the mass matrix

$$
\left(A^{\mu}, B^{\mu}\right)\left(\begin{array}{cc}
g_{c p}^{2} & -g_{c p} g_{F} \\
-g_{c p} g_{F} & g_{F}^{2}
\end{array}\right) V^{2}\left(\begin{array}{c}
A_{\mu} \\
B_{\mu}
\end{array}\right)
$$

which is familiar and diagonalize to

$$
\left(X^{\mu}, G^{\mu}\right)\left(\begin{array}{cc}
g_{c p}^{2}+g_{F}^{2} & 0 \\
0 & 0
\end{array}\right) V^{2}\left(\begin{array}{c}
X_{\mu} \\
G_{\mu}
\end{array}\right)
$$

where

$$
\left(\begin{array}{c}
A^{\mu} \\
B^{\mu}
\end{array}\right)=\left(\begin{array}{cc}
\cos \theta_{F} & -\sin \theta_{F} \\
\sin \theta_{F} & \cos \theta_{F}
\end{array}\right)\left(\begin{array}{c}
G_{\mu} \\
X_{\mu}
\end{array}\right)
$$

with

$$
\sin \theta_{F}=\frac{g_{c p}}{\sqrt{g_{c p}^{2}+g_{F}^{2}}}, \quad \cos \theta_{F}=\frac{g_{F}}{\sqrt{g_{c p}^{2}+g_{F}^{2}}}
$$

Here the $G^{\mu}$ are the gauge bosons of the unbroken $\mathrm{SU}(3)$, which we identify with QCD, and there is a massive color octet of gauge bosons $\left(X^{\mu}\right)$ that also couples to the SM quarks. The low energy QCD coupling, with the standard generator normalization is given by

$$
g_{c}=\frac{g_{F} g_{c p}}{\sqrt{\left(g_{c p}^{2}+g_{F}^{2}\right)}}
$$

Note that this condition implies a minimum value for $\kappa_{F}=g_{F}^{2} / 4 \pi \geq \alpha_{s}$ in order to obtain the physical color coupling. We take $\alpha_{s}(2 \mathrm{TeV}) \simeq 0.09$.

The extra massive octet with mass $M_{F^{\prime}}=\sqrt{g_{c p}^{2}+g_{F}^{2}} V$ couples as

$$
g_{c p} A^{a \mu} \sum_{f} \bar{q}_{f} \gamma_{\mu} T^{a} q_{f} \rightarrow-g_{c} \cot \theta_{F} X^{a \mu} \sum_{f} \bar{q}_{f} \gamma_{\mu} T^{a} q_{f}+\ldots
$$


If we assume that at low energies the massive gauge bosons may be approximated by a NJL model four fermion interaction

$$
\mathcal{L}_{\text {eff }}=-\frac{g_{c}^{2} \cot ^{2} \theta_{F}}{2 ! M_{F^{\prime}}^{2}}\left(\sum_{f} \bar{q}_{f} \gamma_{\mu} T^{a} q_{f}\right)^{2}+\ldots
$$

then the critical couplingf for chiral symmetry breaking in that approximation is

$$
\cot ^{2} \theta_{\text {crit }}=\frac{2 N_{c} \pi}{\left(N_{c}^{2}-1\right) \alpha_{s}(2 \mathrm{TeV})}=26.2
$$

We may now calculate the corrections the model produces to the low energy SM predictions. The universal coloron produces a universal shift to the $Z$ couplings of all the quarks, from (1),

$$
g_{f} \rightarrow g_{f}\left(1+\frac{\alpha_{s} \cot ^{2} \theta M_{Z}^{2}}{6 \pi M_{F^{\prime}}^{2}} \frac{\left(N_{c}^{2}-1\right)}{2 N_{c}} \ln \left(\frac{M_{F^{\prime}}^{2}}{M_{Z}^{2}}\right)\right)
$$

The correction to the coupling of the top quark would have to reflect the large top mass but the top does not enter into $Z$ pole observables. The contribution to the $\mathrm{T}$ parameter in the model is (5)

$$
T=\frac{N_{c}^{2}-1}{2} \frac{3 m_{t}^{4}}{32 \pi^{2} s_{\theta_{w}}^{2} c_{\theta_{w}}^{2} M_{Z}^{2}} \frac{\alpha_{s} \cot ^{2} \theta}{M_{F^{\prime}}^{2}}\left[\ln \left(M_{F^{\prime}}^{2} / m_{t}^{2}\right)\right]^{2}
$$

The model has no $Z^{\prime}$ to mix with the $Z$. Performing a fit to the $Z$-pole data as described in Section 3 produces the $95 \%$ exclusion curve shown in Fig 4 . When the coupling is critical the massive octet must lie above $3 \mathrm{TeV}$ in mass. In Fig 4 we also display the precision bound reported in [24]; that bound comes from the weak $\rho$ parameter constraint $\Delta \rho \leq 0.4 \%$ which assumes the existence of new physics in the S parameter. As there are no large contributions to the $S$ parameter, we derive a stronger bound. These constraints should be compared to the best current experimental limits from collider jet production [24] also shown in Fig 4 the direct search limits are stronger than the precision measurement constraint.

\footnotetext{
${ }^{2}$ Note that, defining the theory in terms of a momentum-space cutoff $\Lambda$, a four fermion interaction $G \bar{\psi} \psi \bar{\psi} \psi$ has a critical coupling $G_{c}=2 \pi^{2} / \Lambda^{2}$ [23]. The smaller value of $\cot \theta_{\text {crit }}$ given in 25, 24] results from using the large- $N$ approximation and ignoring the running in $\alpha_{s}$ from $M_{Z}$ to $M_{F^{\prime}}=\mathcal{O}(2 \mathrm{TeV})$.
} 




Figure 4: Exclusion curves in the $\kappa / \kappa_{c^{-}} M_{F^{\prime}}$ plane for the universal coloron model with a $100 \mathrm{GeV}$ higgs mass. The curve marked [24] is the precision bound from ref. [24]. The D0 curve is the current 95\% confidence level experimental limit from collider jet production, also from [24]. The remaining curves are our fit at the $95 \%$ and $67 \%$ confidence level.

\subsection{Chiral Top Color}

Top color [4] is very similar in the gauge sector to the universal coloron model - above the flavor breaking scale there are two $S U(3)$ gauge groups that break to QCD and a massive color octet of gauge bosons. The SM fermions transform under the weaker ("proto-color group") of the two SU(3) groups except for the left handed top/bottom doublet, $\psi_{L}$, which transforms under the stronger group. The top color group we consider is therefore chiral as in the see-saw model of [6]. The gauge-boson mixing is as for the universal coloron model. Thus below the flavor breaking scale there are the usual SM gluons plus one extra massive octet with mass $M_{F^{\prime}}=\sqrt{g_{c p}^{2}+g_{F}^{2}} V$ and couplings

$g_{c p} A^{a \mu} \bar{\psi}_{L} T^{a} \gamma_{\mu} \psi_{L}+g_{t c} B^{a \mu} \sum_{q} \bar{q} T^{a} \gamma_{\mu} q \rightarrow-g_{c} \cot \theta_{F} X^{a \mu} \bar{\psi}_{L} T^{a} \gamma_{\mu} \psi_{L}+g_{c} \tan \theta_{F} X^{a \mu} \sum_{q} \bar{q} T^{a} \gamma_{\mu} q+\ldots$

where $q$ are any chiral SM quark except the left handed top/bottom multiplet. The critical coupling again corresponds to $\cot ^{2} \theta=26.2$.

The chiral top color model provides universal shifts to the $Z$ couplings of all the quarks, excepting the top/bottom left handed doublet, of the form

$$
g_{q} \rightarrow g_{q}\left(1+\frac{\alpha_{s} \tan ^{2} \theta M_{Z}^{2}}{6 \pi M_{F}^{\prime 2}} \frac{\left(N_{c}^{2}-1\right)}{2 N_{c}} \ln \left(\frac{M_{F^{\prime}}^{2}}{M_{Z}^{2}}\right)\right)
$$


The left handed bottom quark's $Z$ couplings receives the correction (1)

$$
g_{b_{L}} \rightarrow g_{b_{L}}\left(1+\frac{\alpha_{s} \cot ^{2} \theta M_{Z}^{2}}{6 \pi M_{F}^{\prime 2}} \frac{\left(N_{c}^{2}-1\right)}{2 N_{c}} \ln \left(\frac{M_{F^{\prime}}^{2}}{M_{Z}^{2}}\right)\right)
$$

The contribution to the $\mathrm{T}$ parameter in the model is

$$
T=\frac{N_{c}^{2}-1}{2} \frac{m_{t}^{4}}{32 \pi^{2} s_{\theta_{w}}^{2} c_{\theta_{w}}^{2} M_{Z}^{2}} \frac{\alpha_{s}\left(\cot ^{2} \theta+2 \tan ^{2} \theta\right)}{M_{F}^{\prime 2}}\left[\ln \left(M_{F^{\prime}}^{2} / m_{t}^{2}\right)\right]^{2}
$$

The model has no $Z^{\prime}$ that mixes with the $Z$. The result of performing the fit to the EW precision data is shown in Fig 5 and the $95 \%$ confidence limit is relatively low $(1.3 \mathrm{TeV}$ at critical coupling).

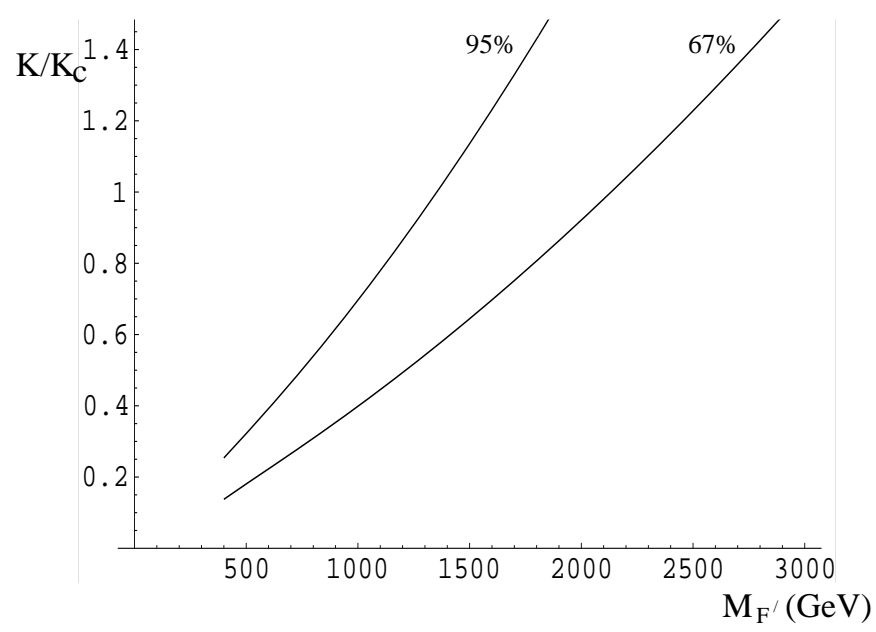

Figure 5: The $95 \%$ and $67 \%$ exclusion curves in the $\kappa / \kappa_{c^{-}} M_{F^{\prime}}$ plane for the chiral top coloron model with a $100 \mathrm{GeV}$ higgs mass.

\subsection{Chiral Quark Family Symmetry}

The gauging of the chiral family symmetry of the left handed quarks has been motivated in technicolor [9], top condensate [5] and flavor universal see saw models [7]. The minimal representative model has a gauged $\mathrm{SU}(3)$ family symmetry, in addition to the SM interactions, acting on the three left handed quark doublets $Q=\left((t, b)_{L}^{i},(c, s)_{L}^{i},(u, d)_{L}^{i}\right)$ where $i$ is a QCD index which commutes with the family symmetry. Note that gauging this symmetry leaves the SM $U(3)^{5}$ global symmetry responsible for the GIM mechanism [8] unbroken and the model is free of tree level FCNC [9]. The additional terms in the lagrangian are

$$
\mathcal{L}=i g_{F} A^{\mu a} \bar{Q} \gamma_{\mu} T^{a} Q
$$


where $T^{a}$ are the generators of $\mathrm{SU}(3)$ corresponding to the three families. We assume that some massive sector induces a vev for an effective higgs that completely breaks the $\mathrm{SU}(3)$ family group giving the gauge bosons masses of order $M_{F}=g_{F} V$ where $V$ is the mass scale associated with the symmetry breaking. There is no mixing with the SM gauge group.

If we assume that at low energies the massive gauge boson may be approximated by a NJL model with coupling $4 \pi \kappa / 2 ! M_{F}^{2}\left(\kappa=g_{F}^{2} / 4 \pi\right)$, then the critical coupling for chiral symmetry breaking in that approximation is

$$
\kappa_{c r i t}=\frac{2 N \pi}{\left(N^{2}-1\right)}=2.36
$$

The chiral quark familons give rise to universal corrections to all the left handed SM quark $Z$ couplings (1). The only subtlety is including the top mass for isospin $+1 / 2$ quarks.

$$
\begin{gathered}
g_{+1 / 2_{L}} \rightarrow g_{+1 / 2_{L}}\left(1+\frac{5 \kappa M_{Z}^{2}}{36 \pi M_{F}^{2}} \ln \left(\frac{M_{F}^{2}}{M_{Z}^{2}}\right)+\frac{\kappa M_{Z}^{2}}{12 \pi M_{F}^{2}} \ln \left(\frac{M_{F}^{2}}{m_{t}^{2}}\right)\right) \\
g_{-1 / 2_{L}} \rightarrow g_{-1 / 2_{L}}\left(1+\frac{2 \kappa M_{Z}^{2}}{9 \pi M_{F}^{2}} \ln \left(\frac{M_{F}^{2}}{M_{Z}^{2}}\right)\right)
\end{gathered}
$$

The contribution to the $\mathrm{T}$ parameter in the model is (5)

$$
T=\frac{N_{c}}{3} \frac{m_{t}^{4}}{32 \pi^{2} s_{\theta_{w}}^{2} c_{\theta_{w}}^{2} M_{Z}^{2}} \frac{\kappa}{M_{F}^{2}}\left[\ln \left(M_{F}^{2} / m_{t}^{2}\right)\right]^{2}
$$

The result of the fit to EW data is shown in Fig 6. At critical coupling the $95 \%$ confidence level bound is approximately $2 \mathrm{TeV}$.

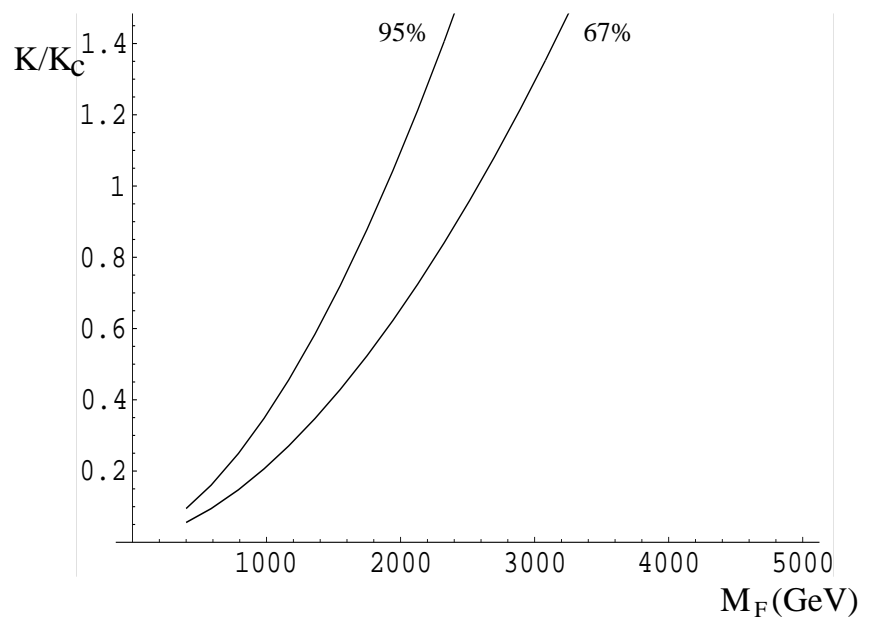

Figure 6: The $95 \%$ and $67 \%$ exclusion curves in the $\kappa / \kappa_{c}-M_{F}$ plane for the gauged, chiral, quark family symmetry model with a $100 \mathrm{GeV}$ higgs mass. 


\subsection{SU(9) Chiral Quark Flavor Symmetry}

We next consider a natural extension of the ideas of gauging the quark family symmetry and chiral color symmetry. That is to gauge the full SU(9) symmetry of both the color and family multiplicity of the left handed quarks. Such a symmetry can be implemented as an extended technicolor gauge symmetry (in the spirit of [13]) or in quark universal seesaw models (as in [7]). Again the SM GIM is preserved [13] The SU(9) symmetry commutes with the standard weak $\mathrm{SU}(2)$ gauge group and acts on the left handed quarks

$$
Q_{L}=\left((t, b)^{r},(t, b)^{b},(t, b)^{g},(c, s)^{r}, \ldots(u, d)^{g}\right)_{L}
$$

with $r, g, b$ the three QCD colors, as

$$
\mathcal{L}=i g_{F} B^{a \mu} \bar{Q}_{L} \Lambda^{a} \gamma_{\mu} Q_{L}
$$

with $\Lambda^{a}$ the generators of $\mathrm{SU}(9)$ which include

$$
\frac{1}{\sqrt{3}}\left(\begin{array}{ccc}
T^{a} & 0 & 0 \\
0 & T^{a} & 0 \\
0 & 0 & T^{a}
\end{array}\right), \frac{1}{\sqrt{6}}\left(\begin{array}{ccc}
T^{a} & 0 & 0 \\
0 & T^{a} & 0 \\
0 & 0 & -2 T^{a}
\end{array}\right), \frac{1}{\sqrt{2}}\left(\begin{array}{ccc}
T^{a} & 0 & 0 \\
0 & -T^{a} & 0 \\
0 & 0 & 0
\end{array}\right)
$$

where $T^{a}$ are the $83 \times 3$ QCD generators. SU(9) further contains

$$
\frac{1}{\sqrt{2}}\left(\begin{array}{ccc}
0 & T^{a} & 0 \\
T^{a} & 0 & 0 \\
0 & 0 & 0
\end{array}\right), \frac{1}{\sqrt{12}}\left(\begin{array}{ccc}
0 & 1 & 0 \\
1 & 0 & 0 \\
0 & 0 & 0
\end{array}\right), \frac{1}{\sqrt{2}}\left(\begin{array}{ccc}
0 & -i T^{a} & 0 \\
i T^{a} & 0 & 0 \\
0 & 0 & 0
\end{array}\right), \frac{1}{\sqrt{12}}\left(\begin{array}{ccc}
0 & -i & 0 \\
i & 0 & 0 \\
0 & 0 & 0
\end{array}\right)
$$

plus the two other similar sets mixing the remaining families. Finally there are two diagonal generators

$$
\frac{1}{\sqrt{12}}\left(\begin{array}{ccc}
1 & 0 & 0 \\
0 & -1 & 0 \\
0 & 0 & 0
\end{array}\right), \frac{1}{\sqrt{36}}\left(\begin{array}{ccc}
1 & 0 & 0 \\
0 & 1 & 0 \\
0 & 0 & -2
\end{array}\right)
$$

The model must also contain the usual interactions of the right handed quarks and leptons. These are included by a proto-color group that acts on the right handed quarks. We normalize the proto-color gauge bosons' couplings such that they have the same generators as the $\mathrm{SU}(9)$ bosons.

$$
\mathcal{L}=\frac{i}{\sqrt{3}} g_{c p} A^{\mu a} \bar{q}_{R} \gamma_{\mu} T^{a} q_{R}
$$

The model must also include the usual SM hypercharge gauge boson.

At the flavor breaking scale we assume an effective higgs, that transforms as a $(9, \overline{9})$ under the $S U(9)_{L} \times S U(9)_{R}$ chiral flavor symmetry, acquires a vev breaking the gauge symmetry 
to a single $\mathrm{SU}(3)$ that becomes QCD. The majority of the $\mathrm{SU}(9)$ gauge bosons have mass $M_{F}=g_{F} V$. Eight of the $\mathrm{SU}(9)$ generators mix with the right handed proto-color group. They correspond to the first generators in (35) which look like the usual QCD interactions of the left handed quarks.

The proto-gluons and flavorons mix through the mass matrix

$$
\left(A^{\mu}, B^{\mu}\right)\left(\begin{array}{cc}
g_{c p}^{2} & -g_{c p} g_{F} \\
-g_{c p} g_{F} & g_{F}^{2}
\end{array}\right) V^{2}\left(\begin{array}{c}
A_{\mu} \\
B_{\mu}
\end{array}\right)
$$

which diagonalizes to

$$
\left(X^{\mu}, G^{\mu}\right)\left(\begin{array}{cc}
g_{c p}^{2}+g_{F}^{2} & 0 \\
0 & 0
\end{array}\right) V^{2}\left(\begin{array}{c}
X_{\mu} \\
G_{\mu}
\end{array}\right)
$$

where

$$
\left(\begin{array}{c}
A^{\mu} \\
B^{\mu}
\end{array}\right)=\left(\begin{array}{cc}
\cos \phi & -\sin \phi \\
\sin \phi & \cos \phi
\end{array}\right)\left(\begin{array}{c}
G_{\mu} \\
X_{\mu}
\end{array}\right)
$$

with

$$
\sin \phi=\frac{g_{c p}}{\sqrt{g_{c p}^{2}+g_{F}^{2}}}, \quad \cos \phi=\frac{g_{F}}{\sqrt{g_{c p}^{2}+g_{F}^{2}}}
$$

The low energy QCD coupling, with the standard generator normalization is given by

$$
g_{c}=\frac{g_{F} g_{c p}}{\sqrt{3\left(g_{c p}^{2}+g_{F}^{2}\right)}}
$$

which implies that $\kappa_{F} \geq 3 \alpha_{s}(2 \mathrm{TeV})$.

Thus the SM fermions' interactions with the massive color octet (with mass $M_{F^{\prime}}=$ $\left.\sqrt{g_{c p}^{2}+g_{F}^{2}} V=M_{F} / c_{\phi}\right)$ are given by

$$
-g_{c} \tan \phi X^{a \mu} \bar{q}_{R} \gamma_{\mu} T^{a} q_{R}+g_{c} \cot \phi X^{a \mu} \bar{q}_{L} \gamma_{\mu} T^{a} q_{L}
$$

If we assume that at low energies the massive gauge bosons may be approximated by a NJL model with coupling $4 \pi \kappa / M_{F}^{2}$ (we ignore the effects of the mixing of eight of the generators with proto-color in this estimate which yields corrections of order $\left.g_{c p} / g_{F}\right)$ ) then the critical coupling for chiral symmetry breaking in that approximation is

$$
\kappa_{c r i t}=\frac{2 N \pi}{\left(N^{2}-1\right)}=0.71
$$

We may now calculate the deviations in low energy parameters in the $\mathrm{SU}(9)$ flavoron theory. The fermion $Z$ vertices are corrected to (1)

$$
\Delta g_{u_{L}}=g_{u_{L}} \frac{\kappa M_{Z}^{2}}{6 \pi M_{F}^{2}}\left[\left(\frac{5 N_{c}}{6}+\frac{c_{\phi}^{4}\left(N_{c}^{2}-1\right)}{6 N_{c}}\right) \ln \left(\frac{M_{F}^{2}}{M_{Z}^{2}}\right)+\frac{N_{c}}{2} \ln \left(\frac{M_{F}^{2}}{m_{t}^{2}}\right)\right]
$$




$$
\begin{aligned}
\Delta g_{u_{R}} & =g_{u_{R}} \frac{\kappa M_{Z}^{2}}{6 \pi M_{F}^{2}}\left[\frac{s_{\phi}^{4}\left(N_{c}^{2}-1\right)}{6 N_{c}}\right] \ln \left(\frac{M_{F}^{2}}{M_{Z}^{2}}\right) \\
\Delta g_{d_{L}} & =g_{d_{L}} \frac{\kappa M_{Z}^{2}}{6 \pi M_{F}^{2}}\left[\frac{4 N_{c}}{3}+\frac{c_{\phi}^{4}\left(N_{c}^{2}-1\right)}{6 N_{c}}\right] \ln \left(\frac{M_{F}^{2}}{M_{Z}^{2}}\right) \\
\Delta g_{d_{R}} & =g_{d_{R}} \frac{\kappa M_{Z}^{2}}{6 \pi M_{F}^{2}}\left[\frac{s_{\phi}^{4}\left(N_{c}^{2}-1\right)}{6 N_{c}}\right] \ln \left(\frac{M_{F}^{2}}{M_{Z}^{2}}\right)
\end{aligned}
$$

where we have neglected the difference between $M_{F}$ and $M_{F^{\prime}}$ in the logarithms.

The contribution to the $\mathrm{T}$ parameter in the model is (5)

$$
T=\frac{m_{t}^{4}}{32 \pi^{2} s_{\theta_{w}}^{2} c_{\theta_{w}}^{2} M_{Z}^{2}} N_{c}\left(\frac{\kappa}{M_{F}^{2}}+\frac{4}{3} \frac{\alpha_{s}\left(\cot ^{2} \phi+2 \tan ^{2} \phi\right)}{M_{F}^{2}}\right)\left[\ln \left(M_{F}^{2} / m_{t}^{2}\right)\right]^{2}
$$

Again there is no mixing with the $Z$ boson.

The results of the fit to the electroweak data are displayed in Fig 7 and the $95 \%$ confidence level limit on the model at its critical coupling is a little below $2 \mathrm{TeV}$.

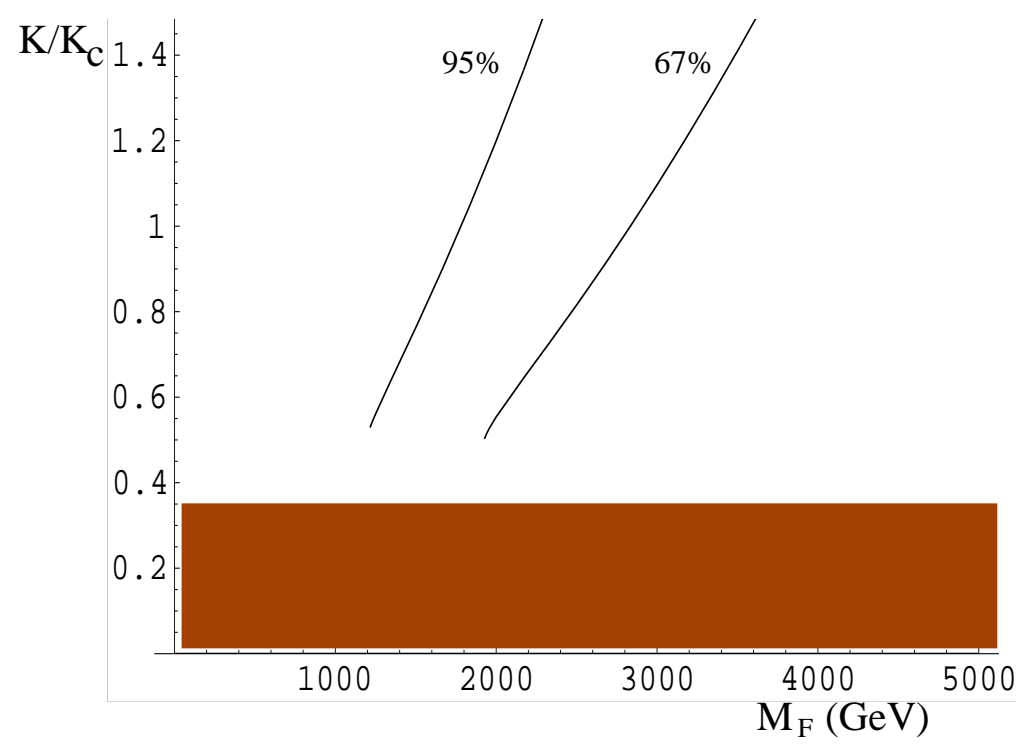

Figure 7: The $95 \%$ and $65 \%$ exclusion curves in the $\kappa / \kappa_{c}-M_{F}$ plane for the gauged chiral quark SU(9) flavor symmetry model with a $100 \mathrm{GeV}$ higgs mass. The shaded region is excluded by the requirement that the measured value of $\alpha_{s}$ is obtained. 


\subsection{SU(12) Chiral Flavor Symmetry}

The final model we consider is one in which we gauge the full SU(12) flavor symmetry of all the left handed SM fermion doublets [13, 7]

$$
Q_{L}=\left((t, b)^{r},(t, b)^{b},(t, b)^{g},\left(\nu_{\tau}, \tau\right),(c, s)^{r}, \ldots\left(\nu_{e}, e\right)\right)_{L}
$$

The flavor gauge interactions act as

$$
\mathcal{L}=i g_{F} B^{a \mu} \bar{Q}_{L} \Lambda^{a} \gamma_{\mu} Q_{L}
$$

with $\Lambda^{a}$ the generators of SU(12), which may be conveniently broken down into the following groupings

$$
\frac{1}{\sqrt{3}}\left(\begin{array}{ccc}
P^{a} & 0 & 0 \\
0 & P^{a} & 0 \\
0 & 0 & P^{a}
\end{array}\right), \frac{1}{\sqrt{6}}\left(\begin{array}{ccc}
P^{a} & 0 & 0 \\
0 & P^{a} & 0 \\
0 & 0 & -2 P^{a}
\end{array}\right), \frac{1}{\sqrt{2}}\left(\begin{array}{ccc}
P^{a} & 0 & 0 \\
0 & -P^{a} & 0 \\
0 & 0 & 0
\end{array}\right)
$$

where $P^{a}$ are the fifteen $4 \times 4$ Pati-Salam generators consisting of eight $3 \times 3$ blocks that correspond to the QCD generators, six step operators between the quarks and leptons and the diagonal generator $1 / \sqrt{24} \operatorname{diag}(1,1,1,-3)$. SU(12) further contains

$$
\frac{1}{\sqrt{2}}\left(\begin{array}{ccc}
0 & P^{a} & 0 \\
P^{a} & 0 & 0 \\
0 & 0 & 0
\end{array}\right), \frac{1}{\sqrt{16}}\left(\begin{array}{ccc}
0 & 1 & 0 \\
1 & 0 & 0 \\
0 & 0 & 0
\end{array}\right), \frac{1}{\sqrt{2}}\left(\begin{array}{ccc}
0 & -i P^{a} & 0 \\
i P^{a} & 0 & 0 \\
0 & 0 & 0
\end{array}\right), \frac{1}{\sqrt{16}}\left(\begin{array}{ccc}
0 & -i & 0 \\
i & 0 & 0 \\
0 & 0 & 0
\end{array}\right)
$$

plus the two other similar sets mixing the remaining families. Finally there are two diagonal generators

$$
\frac{1}{\sqrt{16}}\left(\begin{array}{ccc}
1 & 0 & 0 \\
0 & -1 & 0 \\
0 & 0 & 0
\end{array}\right), \frac{1}{\sqrt{48}}\left(\begin{array}{ccc}
1 & 0 & 0 \\
0 & 1 & 0 \\
0 & 0 & -2
\end{array}\right)
$$

To ensure the SM gauge groups emerge at low energies, we must again introduce a protocolor group as in the SU(9) model above. The first 8 generators of $\mathrm{SU}(12)$ in (53) are the same as those in the $\mathrm{SU}(9)$ model (35) and, hence, the discussion of the mixing between the proto-color and the $8 \mathrm{SU}(12)$ gauge bosons follows the discussion in the $\mathrm{SU}(9)$ model exactly.

In the SU(12) model, though, we must also include a proto-hypercolor gauge boson. The Pati-Salam diagonal generator in the first set of generators in (53) is the traditional generator for the hypercharge boson's coupling to left handed fermions. The proto-hypercharge gauge boson therefore only couples to the right handed fermions. We write the couplings in each 
case in terms of the standard normalized hypercharges $\left(Q=T_{3}+Y\right)$ and with the indicated normalizations of the couplings

$$
\mathcal{L}=i g_{y p} \frac{Y_{R}}{\sqrt{2}} \bar{f}_{R} A^{\mu} f_{R}+i g_{F} \frac{Y_{L}}{\sqrt{2}} \bar{f}_{L} B^{\mu} f_{L}
$$

where $Y_{L}$ and $Y_{R}$ are the left and right handed hypercharges $\left(Y_{L}+Y_{R}=Y\right)$. At the flavor breaking scale the two gauge bosons mix through the mass matrix

$$
\left(A^{\mu}, B^{\mu}\right)\left(\begin{array}{cc}
g_{y p}^{2} & -g_{y p} g_{F} \\
-g_{y p} g_{F} & g_{F}^{2}
\end{array}\right) V^{2}\left(\begin{array}{c}
A_{\mu} \\
B_{\mu}
\end{array}\right)
$$

which diagonalizes to

$$
\left(Z^{\prime} \mu, Y^{\mu}\right)\left(\begin{array}{cc}
g_{y p}^{2}+g_{F}^{2} & 0 \\
0 & 0
\end{array}\right) V^{2}\left(\begin{array}{c}
Z_{\mu}^{\prime} \\
Y_{\mu}
\end{array}\right)
$$

where

$$
\left(\begin{array}{c}
A^{\mu} \\
B^{\mu}
\end{array}\right)=\left(\begin{array}{cc}
\cos \omega & -\sin \omega \\
\sin \omega & \cos \omega
\end{array}\right)\left(\begin{array}{c}
Y_{\mu} \\
Z_{\mu}^{\prime}
\end{array}\right)
$$

with

$$
\sin \omega=\frac{g_{y p}}{\sqrt{g_{y p}^{2}+g_{F}^{2}}}, \quad \cos \omega=\frac{g_{F}}{\sqrt{g_{y p}^{2}+g_{F}^{2}}}
$$

Here $Y^{\mu}$ is the ordinary hypercharge gauge boson.

The low energy hypercharge coupling, with the standard normalization of hypercharges is given by

$$
g_{Y}=\frac{g_{F} g_{y p}}{\sqrt{2\left(g_{y p}^{2}+g_{F}^{2}\right)}}
$$

and $\kappa_{F} \geq \alpha_{Y}(2 \mathrm{TeV}) / 2$ which is a lesser constraint than the constraint from obtaining the correct low energy QCD coupling discussed above.

The massive eigenstate has mass $\sqrt{g_{F}^{2}+g_{y p}^{2}} V=M_{F} / c_{\omega}$ and couples to the SM fermions as

$$
\begin{aligned}
\mathcal{L} & =-i g_{Y} \tan \omega Y_{R} Z^{\prime \mu} \bar{f}_{R} \gamma_{\mu} f_{R}+i g_{Y} \cot \omega Y_{L} Z^{\prime \mu} \bar{f}_{L} \gamma_{\mu} f_{L} \\
& =i \frac{e}{c_{\theta} c_{\omega} s_{\omega}}\left(Y_{L}-s_{\omega}^{2} Y\right) Z^{\prime} \mu \bar{f} \gamma_{\mu} f
\end{aligned}
$$

where we have used the familiar SM result $g_{Y}=e / c_{\theta}$.

If we assume that at low energies the massive gauge boson may be approximated by a NJL model with coupling $4 \pi \kappa / 2 ! M_{F}^{2}$ then the critical coupling for chiral symmetry breaking in that approximation is

$$
\kappa_{\text {crit }}=\frac{2 N \pi}{\left(N^{2}-1\right)}=0.53
$$


Note that combined with the lower bound from the ability to reproduce the QCD coupling $\left(\kappa_{F} \geq 3 \alpha_{s}(2 \mathrm{TeV}) \simeq 0.3\right)$ there is a relatively small window of allowed couplings.

The chiral SU(12) flavoron gives rise to more complicated expressions for the shifts to the $Z$ couplings because it mixes quarks and leptons. The shifts generated by flavoron exchange across the vertices, given by (1), are

$$
\begin{aligned}
& \Delta g_{u_{L}}=g_{u_{L}} \frac{\kappa M_{Z}^{2}}{6 \pi M_{F}^{2}}\left[\left(\frac{5}{2}+\frac{c_{\phi}^{4}\left(N_{c}^{2}-1\right)}{6 N_{c}}+\frac{c_{\omega}^{4} Y_{u_{L}}^{2}}{8}\right) \ln \left(\frac{M_{F}^{2}}{M_{Z}^{2}}\right)\right. \\
& \left.+\frac{N_{c}}{2} \ln \left(\frac{M_{F}^{2}}{m_{t}^{2}}\right)\right]+g_{\nu_{L}} \frac{\kappa M_{Z}^{2}}{6 \pi M_{F}^{2}}\left[\frac{3}{2} \ln \left(\frac{M_{F}^{2}}{M_{Z}^{2}}\right)\right] \\
& \Delta g_{u_{R}}=g_{u_{R}} \frac{\kappa M_{Z}^{2}}{6 \pi M_{F}^{2}}\left[\frac{s_{\phi}^{4}\left(N_{c}^{2}-1\right)}{6 N_{c}}+\frac{s_{\omega}^{4} Y_{u_{R}}^{2}}{8}\right] \ln \left(\frac{M_{F}^{2}}{M_{Z}^{2}}\right) \\
& \Delta g_{d_{L}}=g_{d_{L}} \frac{\kappa M_{Z}^{2}}{6 \pi M_{F}^{2}}\left[4+\frac{c_{\phi}^{4}\left(N_{c}^{2}-1\right)}{6 N_{c}}+\frac{c_{\omega}^{4} Y_{d_{L}}^{2}}{8}\right] \ln \left(\frac{M_{F}^{2}}{M_{Z}^{2}}\right) \\
& +g_{e_{L}} \frac{\kappa M_{Z}^{2}}{6 \pi M_{F}^{2}}\left[\frac{3}{2} \ln \left(M_{F}^{2} / M_{Z}^{2}\right)\right] \\
& \Delta g_{d_{R}}=g_{d_{R}} \frac{\kappa M_{Z}^{2}}{6 \pi M_{F}^{2}}\left[\frac{s_{\phi}^{4}\left(N_{c}^{2}-1\right)}{6 N_{c}}+\frac{s_{\omega}^{4} Y_{d_{R}}^{2}}{8}\right] \ln \left(\frac{M_{F}^{2}}{M_{Z}^{2}}\right) \\
& \Delta g_{\nu_{L}}=g_{\nu_{L}} \frac{\kappa M_{Z}^{2}}{6 \pi M_{F}^{2}}\left[\frac{4}{3}+\frac{c_{\omega}^{4} Y_{\nu_{L}}^{2}}{8}\right] \ln \left(\frac{M_{F}^{2}}{M_{Z}^{2}}\right)+g_{u_{L}} \frac{N_{C} \kappa M_{Z}^{2}}{6 \pi M_{F}^{2}} \ln \left(\frac{M_{F}^{2}}{M_{Z}^{2}}\right) \\
& +g_{u_{L}} \frac{N_{C} \kappa M_{Z}^{2}}{12 \pi M_{F}^{2}} \ln \left(\frac{M_{F}^{2}}{m_{t}^{2}}\right) \\
& \Delta g_{e_{L}}=g_{e_{L}} \frac{\kappa M_{Z}^{2}}{6 \pi M_{F}^{2}}\left[\frac{4}{3}+\frac{c_{\omega}^{4} Y_{e_{L}}^{2}}{8}\right] \ln \left(\frac{M_{F}^{2}}{M_{Z}^{2}}\right)+g_{d_{L}} \frac{3 N_{C} \kappa M_{Z}^{2}}{12 \pi M_{F}^{2}} \ln \left(\frac{M_{F}^{2}}{M_{Z}^{2}}\right) \\
& \Delta g_{e_{R}}=g_{e_{R}} \frac{\kappa M_{Z}^{2}}{6 \pi M_{F}^{2}}\left[\frac{s_{\omega}^{4} Y_{e_{R}}^{2}}{8}\right] \ln \left(\frac{M_{F}^{2}}{M_{Z}^{2}}\right)
\end{aligned}
$$

The contribution to the T parameter from the diagram in Fig 2 in the model is, from (5) $T=\frac{m_{t}^{4}}{32 \pi^{2} s_{\theta_{w}}^{2} c_{\theta_{w}}^{2} M_{Z}^{2}} N_{c}\left(\frac{\kappa}{M_{F}^{2}}+\frac{4}{3} \frac{\alpha_{s}\left(\cot ^{2} \phi+2 \tan ^{2} \phi\right)}{M_{F}^{2}}+\frac{1}{9} \frac{\alpha_{Y}\left(\cot ^{2} \omega+2 \tan ^{2} \omega\right)}{M_{F}^{2}}\right)\left[\ln \left(M_{F}^{2} / m_{t}^{2}\right)\right]^{2}$

Finally we must also include the effects from the mixing between the proto-hypercolor group and the diagonal Pati-Salam generator. We must make some assumption about the structure of the EW breaking sector; we assume that EWS is broken by the equal vevs of a set of fields satisfying $\sum Y_{L}=0$ as would be the case if condensates of all the SM fermions were responsible for the breaking (as in the model of [7]). Writing the standard model $Z$ 
mass as

$$
M_{Z}^{2}=\frac{e^{2}}{s_{\theta}^{2} c_{\theta}^{2}}\left\langle T_{3} T_{3}\right\rangle
$$

where $\left\langle T_{3} T_{3}\right\rangle$ is the expectation value of the EWS breaking operator coupling to the $T_{3}$ currents, the mass mixing between the $Z$ and the $Z^{\prime}$ gauge bosons is given by

$$
\Delta M^{2}=\frac{e^{2}}{s_{\theta} c_{\theta}^{2} s_{\omega} c_{\omega}} s_{\omega}^{2}\left\langle T_{3} T_{3}\right\rangle=\frac{s_{\theta} s_{\omega}}{c_{\omega}} M_{Z}^{2}
$$

where we have used the coupling in (62), the assumption $\sum Y_{L}=0$, and $\left\langle Q T_{3}\right\rangle=0$. The resulting shifts in $Z$ couplings are given by

$$
\delta g_{f}=-\frac{e}{s_{\theta} c_{\theta}} \frac{M_{Z}^{2}}{M_{F}^{2}} s_{\theta}^{2}\left(Y_{L}-s_{\omega}^{2} Y\right)
$$

and the contribution to $\mathrm{T}$ is

$$
T=\frac{s_{\theta}^{2} s_{\omega}^{2}}{\alpha_{E W}} \frac{M_{Z}^{2}}{M_{F}^{2}}
$$

The result of the fit to the EW data is displayed in Fig 8 and the $95 \%$ confidence level limit at critical coupling is $2 \mathrm{TeV}$.

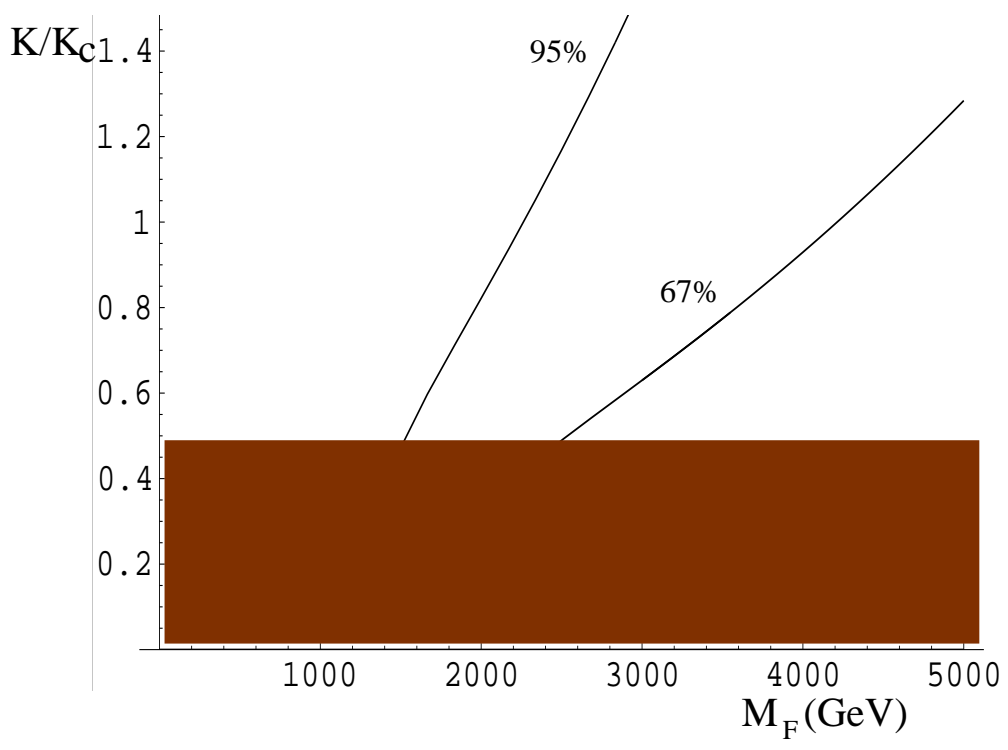

Figure 8: The $95 \%$ and $67 \%$ exclusion curves in the $\kappa / \kappa_{c}-M_{F}$ plane for the gauged chiral $\mathrm{SU}(12)$ flavor symmetry model with a $100 \mathrm{GeV}$ higgs mass. The shaded region is excluded by the requirement that the measured value of $\alpha_{s}$ is obtained. 


\section{Robustness of Bounds}

In this section we address the robustness of the bounds to a number of factors. We will discuss how the fit changes as the higgs mass varies and how strongly the fits depend on the bottom quark asymmetries and left right asymmetry measurement from SLD. Finally the possible influence on the bounds from the presence of additional new physics is investigated using the example of the SM fermions mixing with heavy EW singlet fermions.

\subsection{Variation in the Higgs Mass}

The fits so far performed have assumed a higgs with a mass of $100 \mathrm{GeV}$. It is interesting to study the robustness of the limits to changes in the higgs mass. In fact in many of the dynamical models of EWS breaking we have used to motivate the existence of flavorons, the higgs is expected to be somewhat heavier; typically in flavor universal see-saw models [7] the higgs mass is expected to be of order $400 \mathrm{GeV}$. In the SM such a mass would be excluded but with the additional flavoron physics it is potentially allowed. It is worth noting that the $230 \mathrm{GeV}$ upper bound on the higgs mass in the standard model is largely the result of the SLD measurement of the left right asymmetry. Removing that piece of data raises the limit to of order $400 \mathrm{GeV}$. The influence of the SLD data on the flavoron bounds, however, is small. This can be seen in Fig 9 where the limit on the universal coloron model is shown if the pull of the data from the fit was zero.

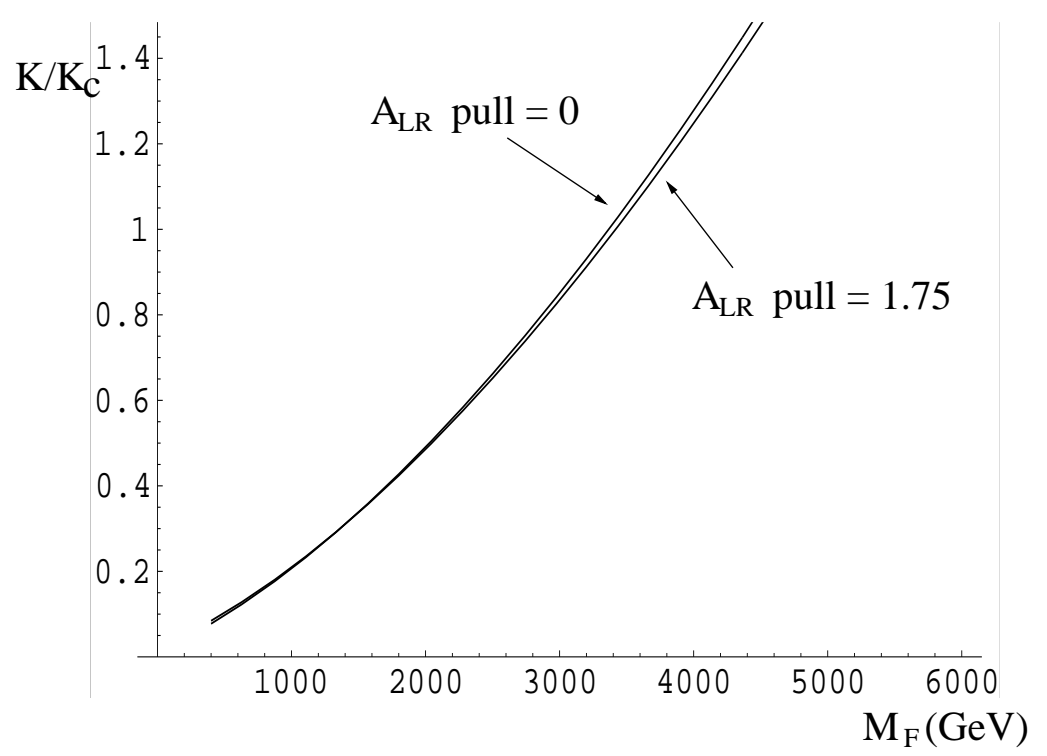


Figure 9: The $95 \%$ exclusion curves in the $\kappa / \kappa_{c}-M_{F}$ plane for the universal coloron model with a $100 \mathrm{GeV}$ higgs mass and varying $A_{L R}$ pull.

As a first example of the higgs mass dependence, we show in Fig 10 the $95 \%$ confidence level bounds on the chiral quark familon model with varying higgs mass. The bound on the flavoron mass scale at critical coupling is relatively insensitive to such changes. The model has little effect on the higgs mass upper bound, which in our fit is $230 \mathrm{GeV}$. The higgs mass dependence of the $\mathrm{SU}(9)$ and $\mathrm{SU}(12)$ chiral flavor symmetry models follow this same pattern as the familon model.

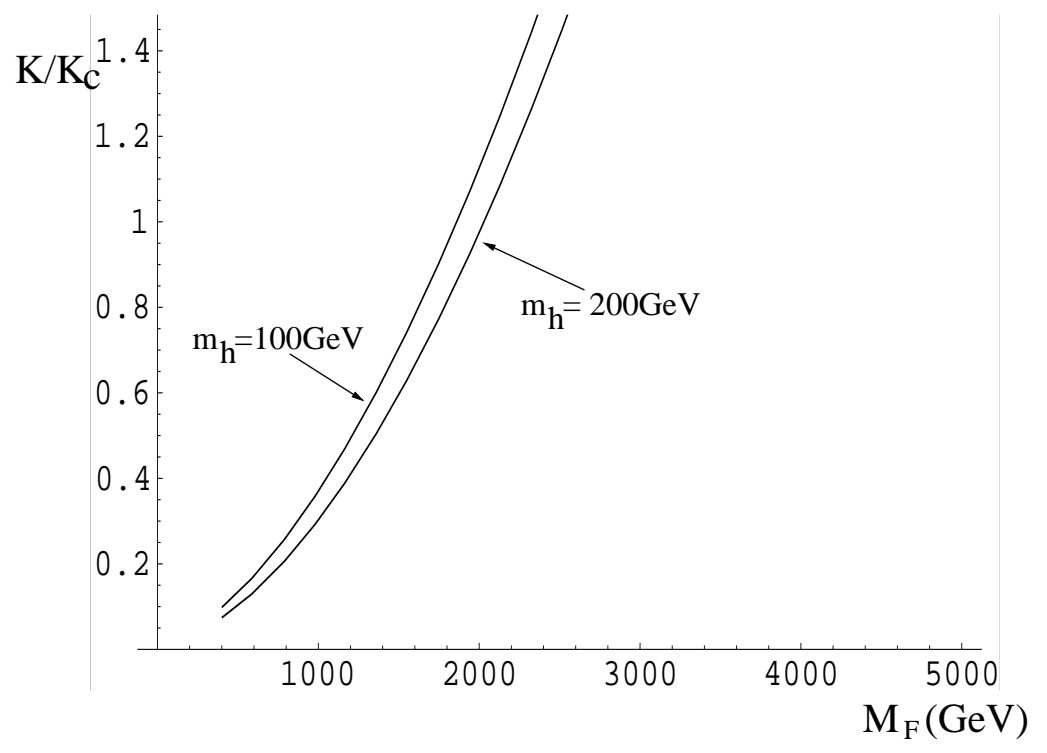

Figure 10: The $95 \%$ exclusion curves in the $\kappa / \kappa_{c}-M_{F}$ plane for the chiral quark family symmetry model with varying higgs mass.

The universal (and chiral top) coloron models have larger contributions to the T parameter which lead to these models' preferring a heavy higgs (which gives negative contributions to T). The dependence on the higgs mass is shown in Fig 11 for the universal coloron model. If the higgs is light then the limits on the colorons rise. For higgs masses above $200 \mathrm{GeV}$ the limits are relatively insensitive. Note that it is possible in these models for the higgs mass to lie above the SM $95 \%$ exclusion limit - indeed, the upper higgs bound can be increased to $650 \mathrm{GeV}$ (or well over $1 \mathrm{TeV}$ if the SLD data point is removed from the fit). Larger higgs masses are only allowed in the presence of extra gauge bosons and there is also a lower bound 
on the coupling of the flavoron at these high higgs mass values. As an example in Fig 12 we show the $95 \%$ exclusion curve for a $400 \mathrm{GeV}$ higgs.

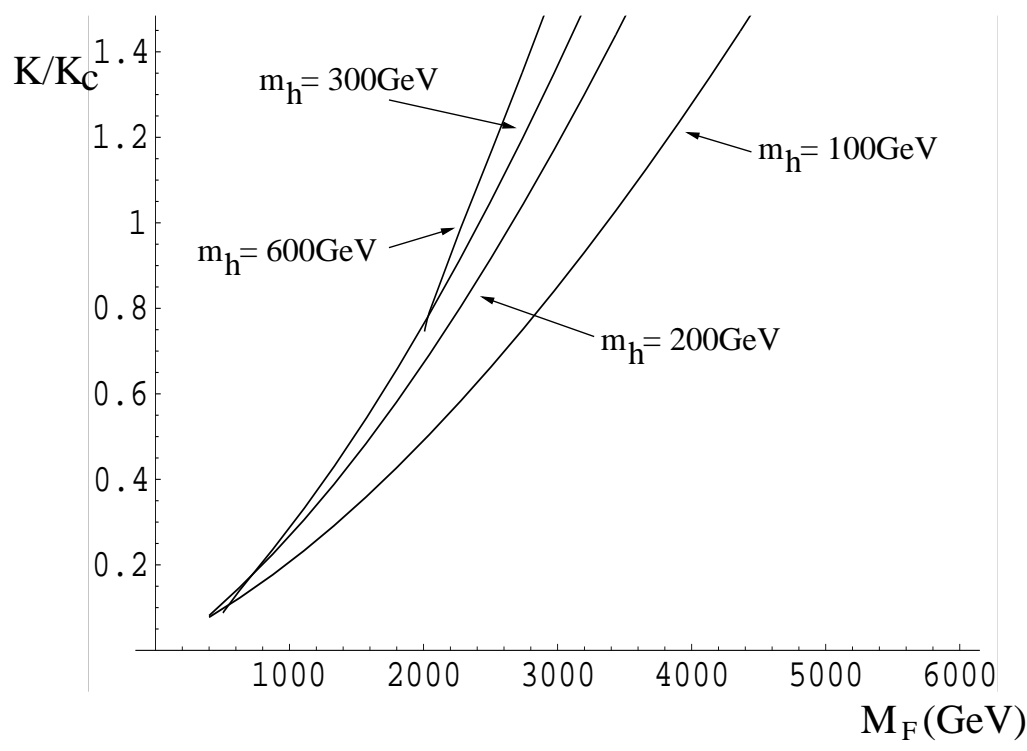

Figure 11: The 95\% exclusion curves from fits to Z-pole data in the $\kappa / \kappa_{c}-M_{F}$ plane for the universal coloron model with varying higgs mass.

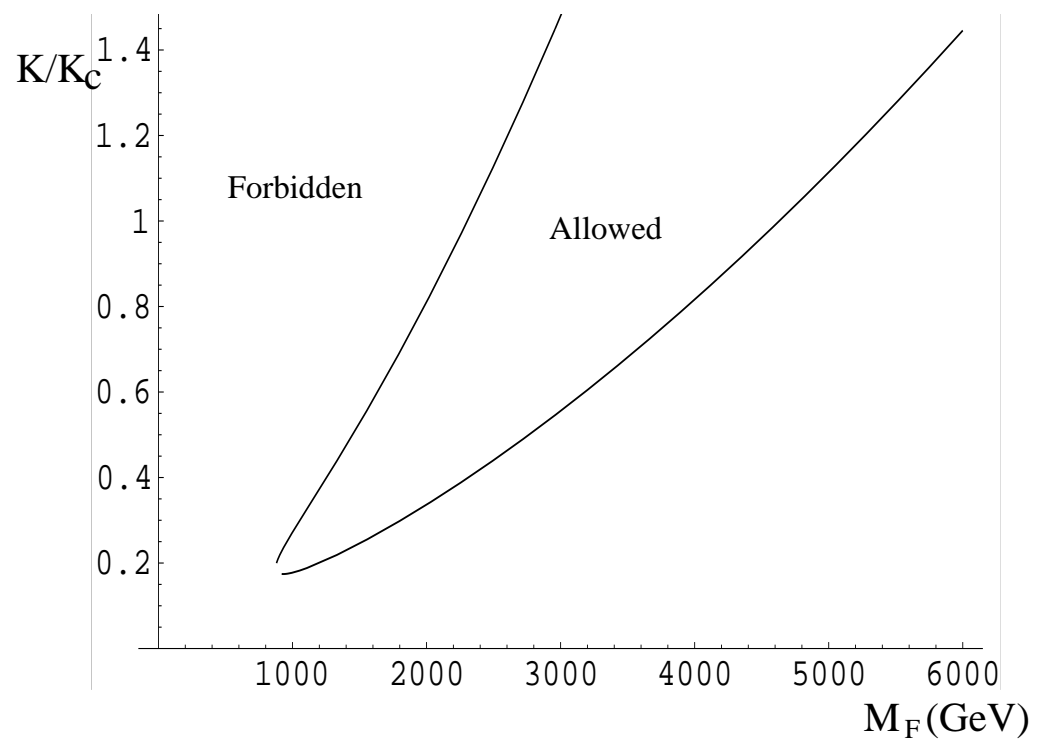


Figure 12: The $95 \%$ exclusion curves in the $\kappa / \kappa_{c}-M_{F}$ plane for the universal coloron model with a $400 \mathrm{GeV}$ higgs mass. Note the appearance of a lower bound on the coloron mass: a $400 \mathrm{GeV}$ higgs is only allowed in the presence of interactions beyond the standard model.

\subsection{The Role of $A_{b}$ and $A_{f b}^{0, b}$}

A cursory overview of the data in (10) reveals that the quantities $A_{b}$ and $A_{f b}^{0, b}$ are those furthest from the SM predictions. What role are these discrepancies playing in the fit? Although the experimental deviation is in the opposite direction to the shifts in these quantities induced by the vertex corrections in (1), their role in constraining the models is not too great. As an indication of the importance of these out lying measurements we show in Fig 13 what the bounds would have been in the universal coloron model if the pulls in the SM fit had been zero. Relaxing these measurements weakens the bound although by only a few hundred $\mathrm{GeV}$. The other flavoron models behave very similarly in this respect to the universal coloron model.

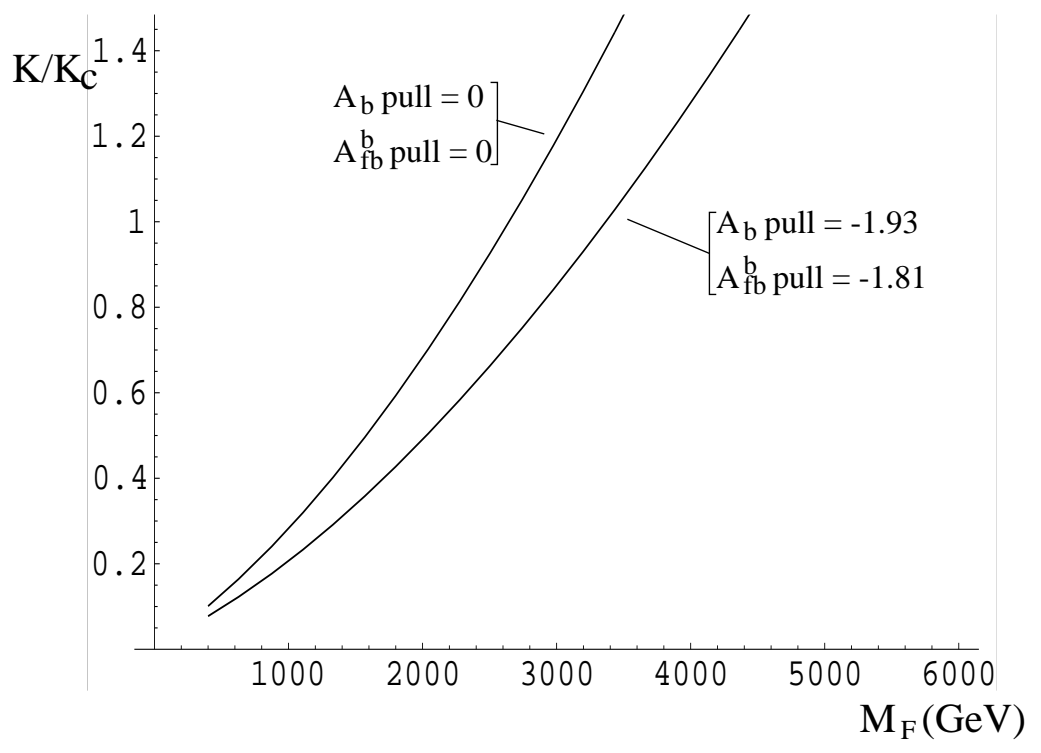

Figure 13: The $95 \%$ exclusion curves in the $\kappa / \kappa_{c^{-}}-M_{F^{\prime}}$ plane for the universal coloron model with a $100 \mathrm{GeV}$ higgs mass and varying $A_{b}$ and $A_{f b}^{b}$ experimental pull. 


\subsection{Flavorons and Fermion Mixing}

As an example of the role that extra new physics can play in changing the bounds on flavorons, in this section we include the possibility of the SM fermions mixing with massive electroweak singlet states. Fermion mixing is an integral part of the top [6] and flavor universal [7] see-saw models. In these models EW symmetry is broken by a condensate between the left handed SM fermions and massive, singlet Dirac fermions with the quantum numbers of the right handed SM fermions. Although these models have a decoupling limit, in which the mass of the EW singlet fermions may be taken arbitrarily high, for the dynamics that breaks EW symmetry to involve the singlets we require that they are not more massive than the flavor gauge bosons.

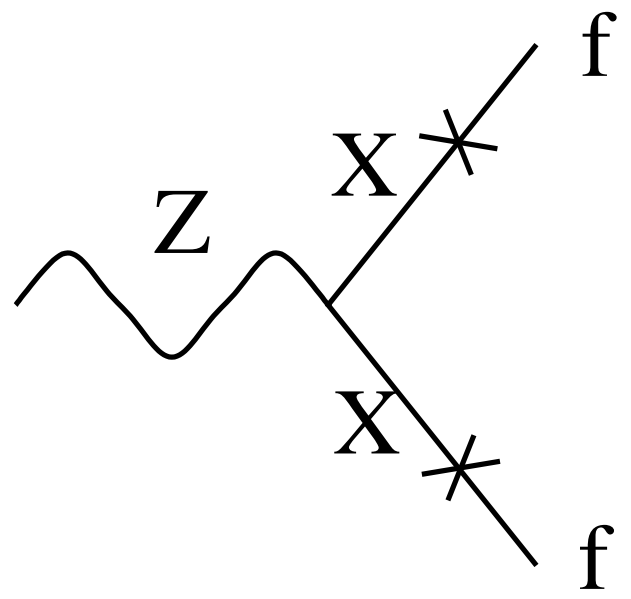

Figure 14: The diagram correcting fermion (f) $Z$ vertices when the SM fermions mix with massive singlets, $\chi$.

To include these effects we assume that all the SM fermions mix with singlets (one pair for each type of massive standard model fermion) with masses $M_{F}$. We assume that the mass mixing term is the same for all the SM fermions and display the results of a fit to the EW data for mass mixings, $M_{m i x}$, between $50 \mathrm{GeV}$ and $150 \mathrm{GeV}$. We treat the mixing in perturbation theory through the graph in Fig 14 so the shifts to the SM fermion $Z$ vertices are

$$
\delta g_{f} \simeq-\frac{e}{s_{\theta} c_{\theta}} Q_{f} s_{\theta_{w}}^{2}\left(\frac{M_{m i x}}{M_{F}}\right)^{2}
$$

since $M_{F} \gg M_{m i x}$.

In Fig 15 we display the results of the fit to the precision data for the chiral, quark familon symmetry model. The fermion mixing gives rise to a sharp cut off in $M_{F}$ where

\footnotetext{
${ }^{3}$ Top-quark mixing with a singlet fermion also effects the one-loop contribution(s) to $\Delta \rho / T$ 6]. This effect can be made relatively small, depending on the details of the model. We include here only the added, and potentially much larger, effect of fermion mixing.
} 
the shifts in the vertices from the mixing alone saturate the experimental bounds. Above that scale the constraints on the flavorons are in fact reduced slightly because the mixing vertex corrections (78) have the opposite sign to those of the flavoron corrections (1)). As the mixing mass rises to $200 \mathrm{GeV}$ the bound from the mixing dominates. The behavior of the familon model is indicative of the effect in the other models where the effect is also small upto the scale of mixing where that new physics dominates the bound.

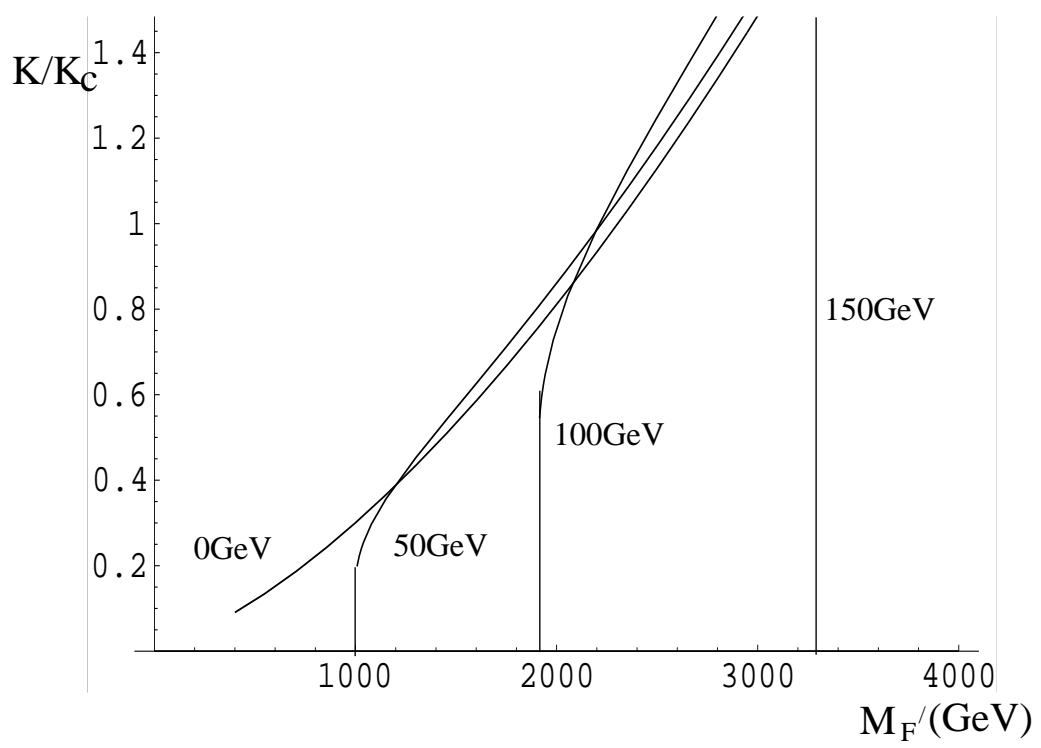

Figure 15: The $95 \%$ exclusion curve in the $\kappa / \kappa_{c}-M_{F}$ plane for the gauged chiral, quark, family symmetry model with universal SM fermion mixing. The four curves correspond to the indicated values of the mixing mass $m_{\operatorname{mix}}\left(\mathbf{8 8 )}\right.$. We take $m_{h}=100 \mathrm{GeV}$.

\section{Conclusions}

Gauged flavor symmetries at low energies have been proposed in models of dynamical electroweak symmetry breaking and fermion mass generation. These massive flavor gauge bosons give rise to corrections to precisely measured electroweak quantities. We have performed fits to the $Z$-pole electroweak data and placed indirect limits on such new physics. In particular we studied several models from the literature: the universal coloron; chiral top color; chiral quark family symmetry; SU(9) and SU(12) chiral flavor symmetry. The $95 \%$ confidence limits on the mass of the heavy gauge bosons for these models, at their critical coupling for chiral symmetry breaking, typically lie between 1 and $3 \mathrm{TeV}$. These limits are more or less insensitive to changes in the higgs mass and the b quark asymmetry data. We have also discussed the inclusion of universal fermion mixing with heavy EW singlets - this effect 
dominates the flavoron bounds when the mixing mass rises above $150 \mathrm{GeV}$. The precision limits are sufficiently low that direct searches at the Tevatron would be expected to be competitive or stronger. Of the models we have studied only the universal coloron's direct search limits have been computed to this point [24] and as we have seen the direct search limits are superior. We will explore the direct search limits in a later paper. 


\section{Acknowledgments}

We thank Bogdan Dobrescu, Chris Hill, and Elizabeth Simmons for comments on the manuscript. This work was supported in part by the Department of Energy under grants DEFG02-91ER40676 and DE-FG02-95ER40896, and by the University of Wisconsin Research Committee with funds granted by the Wisconsin Alumni Research Foundation. NE is grateful for the support of a PPARC Advanced Research Fellowship.

\section{References}

[1] S. Dimopolous and L. Susskind, Nucl. Phys. B155 237 (1979); E. Eitchen and K. Lane, Phys. Lett. B90 125 (1980).

[2] S. Weinberg, Phys. Rev. D19 1277 (1979); L. Suskind, Phys. Rev. D20 2619 (1979); E. Farhi and L. Susskind, Phys. Report 74 No.3 277 (1981).

[3] Y. Nambu, "New Theories In Physics", Proc. XI Warsaw Symposium on Elementary Particle Physics, (ed. Z. Adjuk et al., publ. World Scientific, Singapore, 1989); V.A. Miransky, M.Tanabashi and M. Yamawaki, Phys. Lett. B221 (1989) 177; R.R. Mendel and V.A. Miransky, Phys. Lett. B 268384 (1991); W.A. Bardeen, C.T. Hill and M.Lindner, Phys. Rev. D41 1647 (1990).

[4] C. T. Hill, Phys. Lett. B345, 483 (1995).

[5] S. F. King, Phys. Rev. D45, 990 (1992).

[6] B. Dobrescu and C. T. Hill, Phys. Rev. Lett. 812634 (1998); R.S. Chivukula, B.A. Dobrescu, H. Georgi and C.T. Hill, Phys. Rev. D59 075003 (1999) .

[7] G. Burdman and N. Evans, Phys. Rev. D59 (1999) 115005 and E. H. Simmons, Nucl.Phys. B324 315 (1989).

[8] R. S. Chivukula and H. Georgi, Phys. Lett. 188B 99 (1987).

[9] H. Georgi, "Technicolor and Families", Proc. 1990 International Workshop On Strong Coupling Gauge Theories And Beyond, (ed. T. Muta and K. Yamawaki, publ. World Scientific, Singapore,1991); H. Georgi, Nucl. Phys. B416 699 (1994).

[10] R.S. Chivukula and J. Terning; Phys. Lett. B385 (1996) 209.

[11] D. Karlen, talk at ICHEP 98, Vancouver, B.C., Canada, 23-29 July, 1998 and LEP Electroweak Working Group, CERN-EP/99-15. 
[12] R.S. Chivukula, A.G. Cohen and E.H. Simmons, Phys. Lett. B380 (1996) 92.

[13] L. Randall, Nucl. Phys. B403 122 (1993).

[14] H. Georgi, Nucl. Phys. B156 126 (1979).

[15] R.S. Chivukula, B.A. Dobrescu, and J. Terning, Phys. Lett. B353 (1995) 289.

[16] C.T. Hill and X. Zhang; Phys. Rev. D51 (1995) 3563.

[17] C. Caso et al.; Eur. Phys. J. C3 (1998) 1.

[18] M.E. Peskin and T. Takeuchi, Phys. Rev. Lett. 65964 (1990); M.E. Peskin and T. Takeuchi, Phys. Rev. D46 381 (1992).

[19] R.S. Chivukula, S.B. Selipsky and E.H. Simmons; Phys. Rev. Lett. 69 (1992) 575.

[20] K.Hagiwara, D.Haidt, C.S.Kim and S.Matsumoto, Z. Phys. C64 (1994) 559.

[21] C.P. Burgess, S. Godfrey, H. Konig, D. London and I. Maksymyk, Phys. Rev. D49 (1994) 6115.

[22] B.A. Dobrescu and J. Terning; Phys. Lett. B416 (1998) 129.

[23] Y. Nambu and G. Jona-Lasinio; Phys. Rev. 122 (1961) 345.

[24] I. Bertram and E. H. Simmons, Phys. Lett. B443 347 (1998).

[25] E. H. Simmons, Phys. Rev. D55 1678 (1997). 